\title{
Does the number of metastatic organ involvement affect the response to irinotecan-based chemotherapy in patients with metastatic colorectal
}

\section{cancer?}

\author{
Zedan $\mathrm{A}^{1}$, Khalaf $\mathrm{SM}^{1}$, Hedia $\mathrm{A}^{1}$
}

1 -Department of Medical Oncology, South Egypt Cancer Institute, Assiut University

Correspondence should be addressed to Abdallah Hedia Mohammed at Department of

Medical Oncology, South Egypt Cancer Institute, El-Methak St., Assiut, Egypt,

Abdallah_Elsheref@yahoo.com

published at 21/5/2018

\section{Abstract :}

Background and objectives: Palliative chemotherapy for colorectal cancer(CRC) is more effective than the best supportive care at prolonging survival and improving quality of life. 5-fluorouracil (FU) and leucovorin (LV) modulation were the standard of care, despite having no major impact on survival.In the 1990s, two additional agents, irinotecan and oxaliplatin, were found to have activity against advanced colorectal cancer and have demonstrated survival improvement, when given either alone or in combination with LVIFU, in first- or second-line therapy.

Patients and methods: Prospective study to correlate between response to irinotecan based chemotherapy (FOLFIRI regimen or weekly single agent irinotecan)and the number of metastatic organ involvement in patients with metastatic colorectal cancer as regards responserate and also to compare between FOLFIRI regimen and weekly irinotecan as regards response, survival and toxicity profile in the same patients.

Results: There was significant difference between the two used protocol as regards response whereFOLFIRI arm was superior to weekly single irinotecan arm in patients with 4 or more metastatic involved organs $(P=0.01)$. Toxicity profile was somewhat similar except in alopecia and infection where weekly irinotecan was less incidence of infection and more incidence of alopecia.

Conclusion: FOLFIRI arm was superior to weekly irinotecan arm as regard the response in patient with 4 or more involved organs.

Keyword: FOLFIRI, weekly irinotecan, metastatic CRC, colon, rectum 


\section{Background:}

Colorectal cancer is one of the commonestcancers worldwide. As regards the incidence, CRC considered the third cancer among men comes after prostate and lung cancer and the second among women after breast cancer [1]. CRC represent about $15 \%$ of all cancers and considered the second leading cause of cancer deaths among western countries. About $50 \%$ of CRC patients developed metastatic disease despite adjuvant therapy [2]. Palliative chemotherapy plays a major role in the treatment of colorectal cancer (CRC) patients by improving quality of life and prolonging the survival. 5-Fluorouracil (FU) and Leucovorin (LV) combination were the standard of care for about 40 years in spite of their minimal impact on survival [3]. Oxaliplatin and irinotecan demonstrate remarkable activity against advanced CRC and showed survival improvement, either alone or in combination with 5-FULV [4]. Irinotecan is one of the topoisomerase I inhibitors. Topoisomerase I inhibition leads to double-strand DNA breaks which stimulate cell apoptosis. Addition of irinotecan to 5 FU\LV significantly improves survival in advanced CRC patients [5]. So, US Food and Drug Administration (FDA) approved FOLFIRI regimen as first line therapy in metastatic CRC [4]. In advanced CRC patients progressed after 5-FU based therapy, weekly single agent irinotecan showed promising result either by disease control or improving survival [6]

\section{Patients and Methods:}

\section{Patient population:}

Between May 2013 and May 2017, a total of 52 metastatic CRC patients all of them received oxaloplatin based therapy as first line at the SouthEgypt Cancer Institute were recruited to participate inthis study after approval of the local ethics committeeand patient consent.

Aim of the study: Prospective study to:

1. Correlate between response to irinotecan based chemotherapy (FOLFIRI regimen or weekly single agent irinotecan) and the number of metastatic organ involvement in patients with metastatic colorectal cancer as regards response rate.

2. Compare between FOLFIRI regimen and weekly irinotecan as regards response, survival and toxicity profile in the same patients

\section{Inclusion criteria:}

Patients of both gender, aged $\geq 18$ yearswith histologically confirmed colorectal adenocarcinoma; Patients with documented metastatic colorectal canceraccording to American Joint Committee on Cancer and theInternational Union for Cancer Control (AJCC-UICC); 7th Edition. ECOG performancestate $\leq 2$, adequatehematological, renal andhepatic functions were included in the study.

\section{Exclusion Criteria:}

Patients have been treated with chemotherapy regimens containing irinotecan. Also, patients with inadequate organ functions, and serious uncontrolled concomitant disease that would contraindicate the use of any of the chemotherapy drugs or interfere with cycle's regularity were excluded from the study.

\section{Work-up:}

The routine diagnostic work-up included clinical examination, CT scans of the abdomen, pelvis within 3 weeks before starting treatment, Chest image,blood sampling for complete blood count, renal and hepatic functions. Serum level of tumor marker CEA.

\section{Treatment Schedule:}

26 patients received weekly irinotecan (125 $\mathrm{mg} / \mathrm{m} 2 /$ week intravenous (iv) over 90 minutes for 2 consecutive weeks followed by one week rest) and the other 26 patients received FOLFIRI regimen biweekly (Irinotecan $180 \mathrm{mg} / \mathrm{m} 2$ intravenous day 1, Folinic acid $200 \mathrm{mg} / \mathrm{m} 2$ intravenous day 1 and 2 
before 5-fluorouracil, 5-fluorouracil $400 \mathrm{mg} / \mathrm{m} 2$ iv bolus days 1 and 2 , then $600 \mathrm{mg} / \mathrm{m} 2$ iv 5 -FU infusion over 22 hours, days 1 to 2).Treatmentcontinued until disease progression, unacceptable toxicity or maximum response with maximum 6 cycles. Tumor response measured by the same method of assessment and same technique used to characterize each identified and reported lesion at baseline. Assessment was done every two cycles, accordance to the Response Evaluation Criteria in Solid Tumors (RECIST).Primary end points were overall response rate, progression free survival and side effects profile. Secondary end point was one year overall Survival

\section{Post-treatment follow up:}

History, physical examination, imaging and CEA every 3-6 months for one year.

\section{Quantification of smoking:}

Quantification of smoking was done using the smoking index (SI)[3]. As previously published by the authors, SI was defined as the number of bidis/cigarettes smoked per day multiplied by the number of years smoked. Based upon SI, patients were categorized into the following groups:

\section{Never-smokers \\ II. Light smokers [SI=1-100] \\ III. Moderate smokers [SI=101- 300] \\ IV. Heavy smokers $[\mathrm{SI} \geq 301]$}

\section{Results}

The demographic characteristics of the enrolled patients are shown in Table 1. Mean $( \pm$ SD) age of those patients received irinotecan was $45.09 \pm 12.54$ versus $47.11 \pm 10.34$ years for those received FOLFIRI. We followed the patients until May 2017 with a median follow-up period of 17 months.

The tumor characteristics are shown in Table 2. Rectum was the most frequent affected site with malignancies in the current study. Ten $(38.5 \%)$ patients from irinotecan group versus 8 (30.8\%) patients from FOLFIRI group had rectal carcinoma. Other affected sites in the irinotecan group were

\section{Statistical analysis}

All statistical analyses were performed using SPSS version 20.0 (SPSS Inc., Chicago, IL). Data were described as frequencies (percentages). Differences in distributions between the variables examined were analyzed by chi-square test. PFS was defined as the time from the start of treatment to the time of the first record of progression or to the date of death. OS was assessed as the time from the initiation of first-line chemotherapy $y$ to death from any cause or last follow-up. Survival curve was estimated with the Kaplan-Meier method and compared using the log-rank test. A multivariable Logistic regression analysis included all relevant clinicopathological variables were performed by a forward stepwise method. A P -value $<0.05$ was considered statistically significant

sigmoid colon, ascending colon, descending colon, and transverse colon in $6(23.1 \%), 4(15.4 \%), 4$ $(15.4 \%)$ and $2(7.7 \%)$ patients respectively. Other affected sites in case of FOLFIRI were sigmoid colon, ascending colon, and descending colon in 4 (15.4\%), $4(15.4 \%)$ and $10(38.5 \%)$ patients respectively. adenocarcinoma represent the most common histological type occurred in $18(69.2 \%)$ patients in irinotecan versus 21 patients $(80.8 \%)$ in FOLFIRI group, while mucinous type occurred in 8 patients $(30.8 \%)$ in irinotecan versus 5 patients $(19.2 \%)$ in FOLFIRI group.

As regard site of metastasis, the liver was the commonest single site of metastasis in each group in each group (11 "42.3\%" versus 17 "65.4\%") patients for weekly irinotecan and FOLFIRI group respectively.

In irinotecan group, the number of metastatic organ involved was 2, 3 and 4 organs represent $42.3 \%$, $30.8 \%$ and $26.9 \%$ respectively while in FOLFIRI group, the number of organ involved was 2, 3 and 4 organs represent $38.5 \%, 26.4 \%$ and $34.6 \%$ respectively (Table 3 ). There were no statistically significant differences between both groups regarding distribution of patients as regards site of metastasis and number of involved organs.

As regards relation between the number of metastatic organ involvedand response to irinotecan based chemotherapy, It was noticed that number of metastatic organ involvement had significant effect 
on response to weekly irinotecan regimen in the current study $(\mathrm{P}=0.01)$. Most of patients $(71.4 \%)$ with four organs or more involved in the metastasis had progressive disease while $72.7 \%$ patients with two organs involved had partial response to weekly irinotecan. Fifty percent of those patients with three organs involved had stable disease. But, there is no statistically significant difference in response to FOLFIRI regimen in the current study based on number of metastatic organ involvement.

By inspection of table 4, it was noticed that the toxicity profile of both groups was somewhat similar, except in infection and alopecia.Alopecia occurred in 20 patients in irinotecan group, 13 (50\%) patients of them were G1 while the others were G2. All patients received FOLFIRI developed alopecia, $16(61.5 \%)$ patients of them were G2 while the other were G1 which was statistically significant $(\mathrm{P}$ value $=0.02)$. As regard infection, Grade 1and 2 occurred in $12(46.2 \%)$ and 20 (76.92\%) patients in irinotecan and FOLIFIRI regimen respectively while grade 3 and 4 infection occurred in only one $(3.8 \%)$ patient with irinotecan regimen and two $(7.69 \%)$ patients with FOLFIRI regimen which was statistically significant $(\mathrm{P}$ value $=0.01) \cdot \mathrm{G} 1$ and G2 diarrhea occurred in $17(56.4 \%)$ and $13(50 \%)$ patients in irinotecan and FOLFIRI regimen respectively while G3 and G4 diarrhea occurred in $9(34.6 \%)$ and $13(50 \%)$ patients in irinotecan and FOLFIRI regimen respectively with no statistically significant difference between both group.

As regards response, in our study, partial response, stable disease and progressive disease occurred in 10 (38.5\%), 7 (26.9\%) and $9(34.6 \%)$ patients respectively in weekly irinotecan arm and 8 (30.8\%), 7 (26.9\%) and $11(42.3 \%)$ patients respectively in case of FOLFIRI regimen with no statistically significant difference between two arms (Table 5).

As regards survival, in our study Kaplan Meier survival analysis was used to estimate the overall survival of both groups included in the study.Median survival of all patients included in the study was 10.15 months while median survival of patients who received weekly irinotecan regimen was 10 months versus 8.77 months for those received FOLFIRI regimen with no statistically significant difference between two regimens $(\mathrm{P}=$
0.86). One year survival for weekly irinotecan regimen was $37.4 \%$ versus $36.3 \%$ for FOLFIRI regimen (Figure 1).In our study, it was noticed that $19(73.08 \%)$ patients received FOLFIRI regimen had progressive disease and median time to disease progression was 11 months while 22 (84.6\%) patients had progressive disease in case of irinotecan regimen with median time to disease progression was 8.8 months, with no statistically significant difference $(\mathrm{P}=0.56)$ (Figure 2$)$.

\section{Discussion}

Colorectal cancer is the third commonly diagnosed cancer worldwide. CRC affect men and women of all racial and ethnic groups. CRC accounts for $10 \%$ to $15 \%$ of all cancers and it is the second leading cause of cancer deaths in western countries. Approximately half of all colorectal cancer patients develop metastatic disease[2].According to FDA approval in March 2000, FOLFIRI regimen considered one of the standard first-line therapies for advanced colorectal cancer to which new regimens should be compared[5]. A weekly irinotecan administration can induce tumor control in patients with advanced colorectal cancer that has progressed during or shortly after 5-fluorouracil based chemotherapy [6].

As regards non-hematological toxicity, in our study grade (I-II) nausea and vomiting occurred in 22 $(84.6 \%)$ patients who received weekly irinotecan and occurred in $21(80.8 \%)$ patients who received FOLFIRI regimen. While grade (III-IV) occurred in $4(15.4 \%)$ patients in weekly irinotecan group and 5 (19.2\%) patients of FOLFIRI group with no statistically significant difference between two groups (Pvalue $=0.13$ ). These data are in contrary with data shown in Clark et al, 2012[10] where grade (III-IV) vomiting occurred more common with single agent irinotecan (19\%) than with FOLFIRI regimen (10\%).Grade III-IV diarrhea occurred in $6(23.07 \%)$ patients in the weekly irinotecan arm and $8(30.7 \%)$ patients in the FOLFIRI arm with no statistically significant difference between two arms $(P$ value $=0.71)$. Consistent with our results, Fuchs et al, 2003[11]demonstrated that grade (III-IV) diarrhea occurred in $36 \%$ of patients received weekly irinotecan. 
As regards hematological toxicity, in our study, grade (I-II) anemia occurred in $8(30.76 \%)$ patients who received weekly irinotecan and 14 (53.84\%) patients who received FOLFIRI regimen while, grade (III-IV) anemia occurred in four $(15.38 \%)$ patients who received weekly irinotecan and five (19.23\%) patients who received FOLFIRI regimen with no statistically significant difference between two groups (Pvalue $=0.32$ ). In contrary, Rosati et al,2006[7]reported that grade (I-II) anemia occurred in $78 \%$ while grade (III-IV) occurred in $8.6 \%$ of patients received weekly irinotecan.Grade (III-IV) neutropenia occurred in only one patient $(3.8 \%)$ of weekly irinotecan arm and in three patients $(11.5 \%)$ of FOLFIRI arm with no statistically significant difference between two arms ( $\mathrm{P}$ value = 0.63).Comparable results have been reported by Clarke et al,2012[10]where grade (III-IV) neutropenia occurred in $5 \%$ of patients in weekly arm and $14 \%$ of FOLFIRI arm.

As regards infection, Grade (I-II) infection occurred in $12(46.2 \%)$ patients in weekly irinotecan arm and $20(76.92 \%)$ patients of FOLFIRI arm while grade (III-IV) occurred only in one $(3.8 \%)$ patient in weekly irinotecan arm and two $(7.69 \%)$ patients in FOLFIRI arm which was statistically significant (P value $=0.01)$. These data are consistent with data shown in Schoemaker et al, 2004[4]where grade (III-IV) infection occurred in $5 \%$ of patients who received weekly irinotecan.

As regards alopecia, in our study, grade I-II occurred in $13(50 \%)$ patients in weekly irinotecan arm and $10(38.5 \%)$ patients with FOLFIRI arm which was statistically significant $(P$ value $=0.02)$. These data are somewhat similar to data observed in Rosati et al,2006[7] where grade (I-II) alopecia occurred in $47.7 \%$ of patients received weekly irinotecan.

As regards response, in our study, partial response, stable disease and progressive disease occurred in $10(38.5 \%), 7(26.9 \%)$ and $9(34.6 \%)$ patients respectively in weekly irinotecan arm and 8 (30.8\%), 7 (26.9\%), and $11(42.3 \%)$ patients respectively in case of FOLFIRI regimen with no statistically significant difference between two arms. These data are in contrary with data observed in Schoemaker et al,2004[4]where partial response, stable disease and progressive disease occurred in $5 \%, 62 \%$ and $19 \%$ in patient received weekly irinotecan respectively.

\section{A significant relation between the number of metastatic organ involvement} and response to irinotecan based chemotherapy was noticed in our study.

It was noticed that number of metastatic organ involvement had significant effect on response to weekly irinotecan regimen in the current study ( $P$ value $=0.01)$. Most of patients $(71.4 \%)$ with four organs or more involved in the metastasis had progressive disease while $72.7 \%$ patients with two organs involved had partial response to weekly irinotecan. Fifty percent of those patients with three organs involved had stable disease. But, there is no statistically significant difference in response to FOLFIRI regimen in the current study based on number of metastatic organ involvement. These data are somewhat similar to data observed at Saltz et al, 2000[6] where combination regimen was better response rate than single agent regimen in patients with $>2$ site of metastasis.

As regards survival, in our study Kaplan Meier survival analysis was used to estimate the overall survival of both groups included in the study. Median survival of all patients included in the study was 10.15 months while median survival of patients who received weekly irinotecan regimen was 10 months versus 8.77 months for those received FOLFIRI regimen with no statistically significant difference between two regimens $(P$ value $=0.86)$. One year survival for weekly irinotecan regimen was $37.4 \%$ versus $36.3 \%$ for FOLFIRI regimen.As regards weekly irinotecan arm, these data are somewhat similar to data shown in Saltz et al, 2000[6]where median survival in patients who received weekly irinotecan was 11 months. As regards FOLFIRI arm, our data are in contrary with data shown in Colucci et al, 2005[12]where median survival in patients received FOLFIRI was 14 months.

In our study, it was noticed that $19(73.08 \%)$ patients received FOLFIRI regimen had progressive disease and median time to disease progression was 11 months while $22(84.6 \%)$ patients had progressive disease in case of irinotecan regimen with median time to disease progression was 8.8 months, with no statistically significant difference 
$(P$ value $=0.56)$.Ourdata are better than these data shown in Saltz et al,2000[6]where median TTP was 4.2 months in weekly single agent irinotecan and 7 months in combination arm. And also, better than data shown in Golucci et al,2005[11] where median TTP was 7 months in patients who received FOLFIRI regimen.

In summary: Weekly single agent irinotecan was not inferior to FOLFIRI regimen as regard response and survival except in patients with 4 or more metastatic involved organs where single agent irinotecan showed poor response rate.

\section{References}

1. Jemal A, Ward EM, Johnson CJ:Annual Report to the Nation on the Status of Cancer, 1975-2014, Featuring Survival.J Natl Cancer Inst. 2017 Sep 1;109(9).

2. Ahmed G, Basel E, Aida A: Alexandria Journal of Medicine 2014; 50: 197-201

3. Singh N, Aggarwal AN, Gupta D: Prevalence of low body mass index among newly diagnosed lung cancer patients in North India and its association with smoking status. Thoracic Cancer 2011;2:27-31.

4. Schoemaker N, Kuppens I, Moiseyenko V: A randomised phase II multicentre trial of irinotecan (CPT-11) using four different schedules in patients with metastatic colorectal cancer. British Journal of Cancer. 2004; 91(8):1434-1441.

5. Douillard JY, Cunningham D, Roth AD: Irinotecan combined with fluorouracil compared with fluorouracil alone as

13. theGruppoOncologicoDell'ItaliaMeridio nale. J ClinOncol 2005;23:4866-4875. first-line treatment for metastatic colorectal cancer;Amulticentrerandomised trial. The Lancet 2000; 355:1041-1047.

6. Saltz LB, Cox JV, Blanke C: Irinotecan plus fluorouracil and leucovorin for metastatic colorectal cancer. N Engl J Med 2000; 343(13):905-914.

7. RosatiG, and Cordio S: Single-agent irinotecan as second-line weekly chemotherapy in advanced colorectal cancer.Tumori; 2006;92(4):290-4.

8. Abou-zeid A.A, Khafagy W, Marzouk D.M: Colorectal Cancer in Egypt. Dis. Colon. Rectum, Sep., 45 (9): 1255-60, 2002.

9. Veruttipong D, Soliman AS, Gilbert SF: Age distribution, polyps and rectal cancer in the Egyptian population-based cancer registry. World J Gastroenterol 2012; 18(30): 3997-4003

10. Clarke S, Yip S, Brown C, van Hazel G: Single-agent irinotecan or FOLFIRI as second-line chemotherapy for advanced colorectal cancer; results of a randomised phase II study (DaVINCI) and meta-analysis. European Journal of Cancer 2012; 48(3): 407.

11. FuchsCS, Moore MR, Harker G: Phase III comparison of two irinotecan dosing regimens in second-line therapy of metastatic colorectal cancer. J ClinOncol, 2003; 21: 807-814.

12. Colucci G,Gebbia V, Paoletti G: Phase III randomized trial of FOLFIRI versus FOLFOX4 in the treatment of advanced colorectal cancer: a multicenter study of

\section{List of Figures:}

Figure 1:Kaplan Meier survival analysis in our study.

Figure 2:Progression free survival in both regimens

\section{List of Tables:}

Table1:The demographic characteristics of the 53 colorectal cancer patients in the study.

Table 2:Tumor characteristics of the colorectal cancer in studied patients. 
Table 3:Number of metastatic organ involvement

Table 4:Adverse effects of chemotherapy in both groups.

Table 5 :Outcome of the studied patients.

\begin{tabular}{|c|c|c|c|}
\hline Variables & Group I $(\mathrm{N}=26)(\%)$ & $\begin{array}{l}\text { Group II } \quad(\mathrm{N}=26) \\
(\%)\end{array}$ & $P$ value \\
\hline Age (years) & $45.09 \pm 12.54$ & $47.11 \pm 10.34$ & 0.31 \\
\hline $\begin{array}{l}\text { Sex } \\
\text { Male } \\
\text { Female }\end{array}$ & $\begin{array}{l}13(50) \\
13(50)\end{array}$ & $\begin{array}{l}15(57.7) \\
11(42.3)\end{array}$ & 0.39 \\
\hline $\begin{array}{c}\text { Residence } \\
\text { Urban } \\
\text { Rural }\end{array}$ & $\begin{array}{l}1(3.8) \\
25(96.2)\end{array}$ & $\begin{array}{l}6(23.1) \\
20(76.9)\end{array}$ & 0.06 \\
\hline $\begin{array}{l}\text { Smoking } \\
\text { Non smoker } \\
\text { Light smoker }(\mathrm{SI}=1-100) \\
\text { Moderate smoker }(\mathrm{SI}=101-300) \\
\text { Heavy smoker }(\mathrm{SI} \geq 301)\end{array}$ & $\begin{array}{l}14(53.8) \\
0 \\
4(15.4) \\
8(30.8)\end{array}$ & $\begin{array}{l}12(46.2) \\
1(3.8) \\
5(19.2) \\
8(30.8)\end{array}$ & 0.73 \\
\hline $\begin{array}{l}\text { Response to the previous line of } \\
\text { chemotherapy in adjuvant sitting: } \\
\text { Chemosensitive } \\
\text { Chemoresistant }\end{array}$ & $\begin{array}{l}9(34.6) \\
17(65.4)\end{array}$ & $\begin{array}{l}15(57.7) \\
11(42.3) \\
\end{array}$ & 0.08 \\
\hline
\end{tabular}

Table 1: Demographic data of studied patients

\begin{tabular}{|c|c|c|c|}
\hline Variables & $\begin{array}{l}\text { Weekly } \\
\text { irinotecan } \\
(\mathbf{n = 2 6})\end{array}$ & $\begin{array}{l}\text { FOLFIRI } \\
(n=26)\end{array}$ & P value \\
\hline $\begin{array}{l}\text { Site } \\
\text { Rectum } \\
\text { Sigmoid } \\
\text { Ascending colon } \\
\text { Transverse colon } \\
\text { Descending colon } \\
\end{array}$ & $\begin{array}{l}10(38.5) \\
6(23.1) \\
4(15.4) \\
2(7.7) \\
4(15.4)\end{array}$ & $\begin{array}{l}8(30.8) \\
4(15.4) \\
4(15.4) \\
0(0.0) \\
10(38.5)\end{array}$ & 0.26 \\
\hline $\begin{array}{l}\text { Histological type } \\
\text { Adenocarcinoma } \\
\text { Mucinous adenocarcinoma }\end{array}$ & $\begin{array}{l}18(69.2) \\
8(30.8)\end{array}$ & $\begin{array}{l}21(80.8) \\
5(19.2)\end{array}$ & 0.27 \\
\hline $\begin{array}{l}\text { Grade } \\
\text { II } \\
\text { III } \\
\end{array}$ & $\begin{array}{l}18(69.2) \\
8(30.8)\end{array}$ & $\begin{array}{l}13(50) \\
13(50)\end{array}$ & 0.13 \\
\hline $\begin{array}{l}\text { Stage at diagnosis } \\
\text { III } \\
\text { IV }\end{array}$ & $\begin{array}{l}22(84.6) \\
4(15.4)\end{array}$ & $\begin{array}{l}21(80.8) \\
5(19.2)\end{array}$ & 0.50 \\
\hline
\end{tabular}

Table 2: Tumor characteristics of the colorectal cancer in studed patients 


\begin{tabular}{|l|l|l|l|}
\hline $\begin{array}{l}\text { Number of metastatic } \\
\text { organ involvement }\end{array}$ & $\begin{array}{l}\text { Group I (n= } \\
\text { 26) }\end{array}$ & $\begin{array}{l}\text { Group II (N= } \\
\text { 26) }\end{array}$ & $\begin{array}{l}\boldsymbol{P} \\
\text { value }\end{array}$ \\
\hline$\leq 2$ & $11(42.3)$ & $10(38.5)$ & 0.42 \\
3 & $8(30.8)$ & $7(26.9)$ & \\
4 & $7(26.9)$ & $9(34.6)$ & \\
\hline \\
Data was expressed in form of frequency (percentage). $P$ value was significant if < \\
0.05
\end{tabular}

Table 3: Number of metastatic organ involvement

\begin{tabular}{|c|c|c|c|}
\hline Variables & $\begin{array}{l}\text { Weekly } \\
\text { irinotecan } \\
(\mathbf{n}=\mathbf{2 6})\end{array}$ & $\begin{array}{l}\text { FOLFIRI } \\
(n=26)\end{array}$ & P value \\
\hline $\begin{array}{c}\text { Nausea \& Vomiting } \\
\text { G1,2 } \\
\text { G3,4 }\end{array}$ & $\begin{array}{l}22(84.6) \\
4(15.4)\end{array}$ & $\begin{array}{l}21(80.8) \\
5(19.2)\end{array}$ & 0.13 \\
\hline $\begin{array}{r}\text { Diarrhea } \\
\text { G1,2 } \\
\text { G3,4 } \\
\end{array}$ & $\begin{array}{l}20(76.93) \\
6(23.07)\end{array}$ & $\begin{array}{l}18(69.3) \\
8(30.7)\end{array}$ & 0.71 \\
\hline $\begin{array}{c}\text { Mucositis } \\
\text { G1,2 } \\
\text { G3,4 } \\
\end{array}$ & $\begin{array}{l}19(73.1) \\
7(26.9)\end{array}$ & $\begin{array}{l}18(69.2) \\
8(30.8)\end{array}$ & 0.63 \\
\hline $\begin{array}{l}\text { Fever } \\
\text { No } \\
\text { G1,2 } \\
\end{array}$ & $\begin{array}{l}6(23.1) \\
20(76.9) \\
\end{array}$ & $\begin{array}{l}4(15.4) \\
22(84.6) \\
\end{array}$ & 0.46 \\
\hline $\begin{array}{l}\text { Hepatoxicity } \\
\text { No } \\
\text { G1,2 } \\
\text { G3,4 }\end{array}$ & $\begin{array}{l}15(57.7) \\
7(26.9) \\
4(15.4) \\
\end{array}$ & $\begin{array}{l}20(76.92) \\
4(15.38) \\
2(7.69) \\
\end{array}$ & 0.67 \\
\hline $\begin{array}{c}\text { Bleeding } \\
\text { No } \\
\text { G1,2 } \\
\text { G3,4 } \\
\end{array}$ & $\begin{array}{l}21(80.8) \\
1(3.8) \\
4(15.4) \\
\end{array}$ & $\begin{array}{l}21(80.8) \\
4(15.4) \\
1(3.8) \\
\end{array}$ & 0.36 \\
\hline $\begin{array}{c}\text { Infection } \\
\text { No } \\
\text { G1,2 } \\
\text { G3,4 } \\
\end{array}$ & $\begin{array}{l}13(50) \\
12(46.2) \\
1(3.8) \\
\end{array}$ & $\begin{array}{l}4(15.38) \\
20(76.92) \\
2(7.69) \\
\end{array}$ & 0.01 \\
\hline $\begin{array}{c}\text { Alopecia } \\
\text { No } \\
\text { G1 } \\
\text { G2 } \\
\end{array}$ & $\begin{array}{l}6(23.1) \\
13(50) \\
7(26.9) \\
\end{array}$ & $\begin{array}{l}0 \\
10(38.5) \\
16(61.5) \\
\end{array}$ & 0.02 \\
\hline $\begin{array}{c}\text { Anemia } \\
\text { No } \\
\text { G1,2 } \\
\text { G3,4 } \\
\end{array}$ & $\begin{array}{l}14(53.84) \\
8(30.76) \\
4(15.38) \\
\end{array}$ & $\begin{array}{l}7(26.92) \\
14(53.84) \\
5(19.23) \\
\end{array}$ & 0.32 \\
\hline $\begin{array}{c}\text { Neutropenia } \\
\text { No } \\
\text { G1,2 } \\
\text { G3,4 } \\
\end{array}$ & $\begin{array}{l}3(11.5) \\
22(84.6) \\
1(3.8) \\
\end{array}$ & $\begin{array}{l}4(15.4) \\
19(73.1) \\
3(11.5) \\
\end{array}$ & 0.63 \\
\hline $\begin{array}{l}\text { Thrombocytopenia } \\
\text { No } \\
\text { G1,2 } \\
\text { G3,4 } \\
\end{array}$ & $\begin{array}{l}4(15.4) \\
18(69.2) \\
4(15.4) \\
\end{array}$ & $\begin{array}{l}0 \\
25(96.2) \\
1(3.8) \\
\end{array}$ & 0.06 \\
\hline
\end{tabular}

Data was expressed in form of frequency (percentage). P value was significant if $<0.05$

Table 4: Adverse Effects of Chemotherapy in both Groups 


\begin{tabular}{|l|l|l|l|}
\hline Variables & $\begin{array}{l}\text { Weekly } \\
\text { irinotecan } \\
(\mathbf{n = 2 6})\end{array}$ & $\begin{array}{l}\text { FOLFIRI } \\
(\mathbf{n = 2 6 )}\end{array}$ & P value \\
\hline Dose modification & $12(46.2)$ & $14(53.8)$ & 0.93 \\
\hline Response & $10(38.5)$ & $8(30.8)$ & 0.81 \\
Partial response & $7(26.9)$ & $7(26.9)$ & \\
Stable disease & $9(34.6)$ & $11(42.3)$ & \\
$\quad$ Progressive disease & $17(65.4)$ & $15(57.7)$ & 0.09 \\
Clinical benefit** & & & \\
\hline
\end{tabular}

Table 5: Outcome of the studied patients

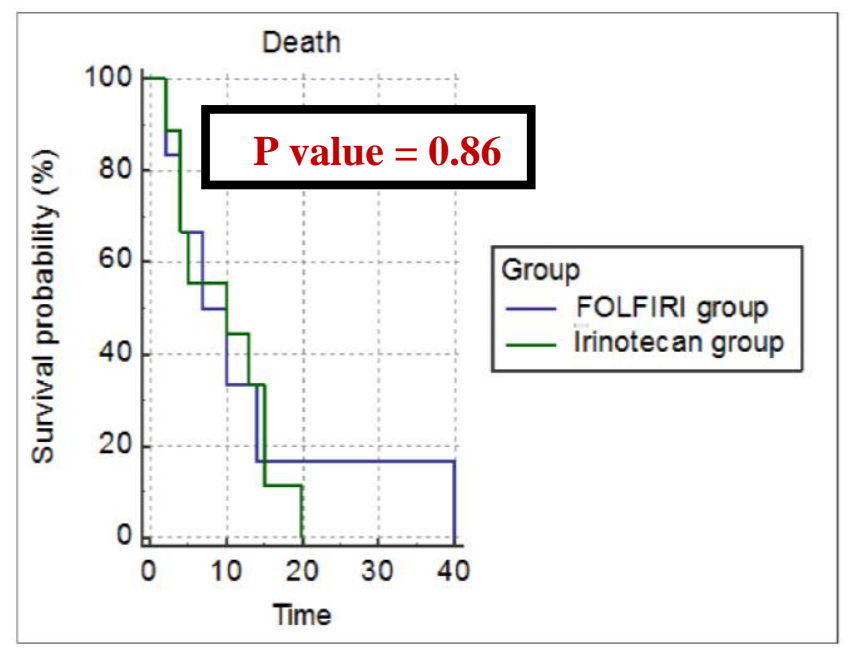

Figure 1: Median survival difference between FOLFIRI arm and weekly irinotecan arm of patients in our study by Kaplan Meier Survival Analysis.

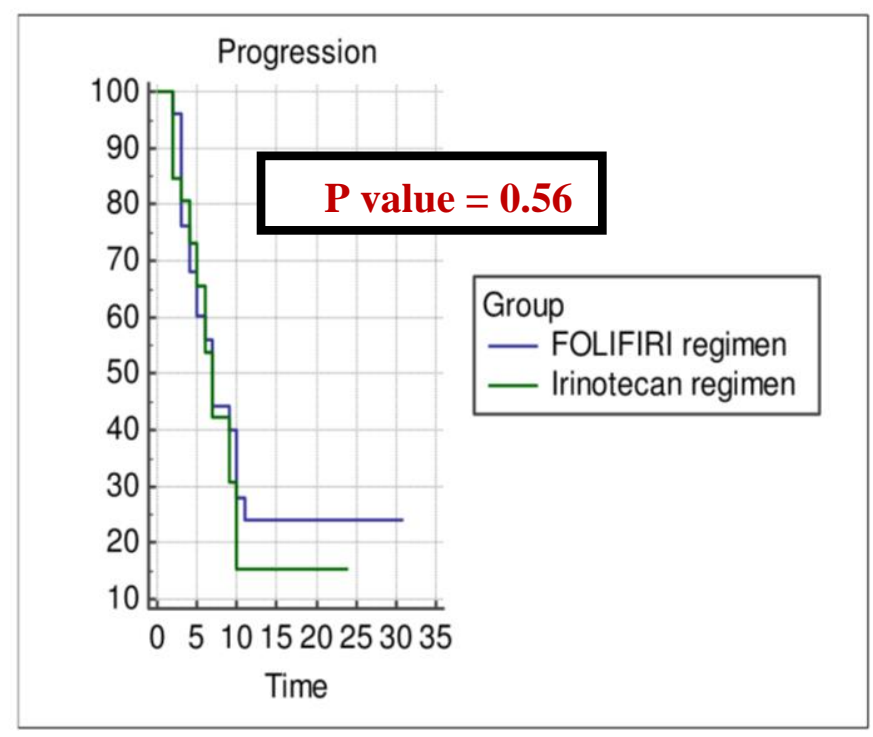

Figure 2: Progression free survival difference between FOLFIRI arm and weekly irinotecan arm of patients in our study by Kaplan Meier Survival Analysis. 